\title{
A review of threshold time series models in finance
}

\author{
Cathy W. S. Chen*, Mike K. P. So and Feng-Chi Liu
}

Since the pioneering work by Tong $(1978,1983)$, threshold time series modelling and its applications have become increasingly important for research in economics and finance. A number of books and a vast number of research papers published in this area have been motivated by Tong's threshold models. The goal of this paper is to give a through review on the development of the family of threshold time series model in finance and to provide a streamlined approach to financial time series analysis. It covers threshold modeling, nonlinearity tests, statistical inference, diagnostic checking, and model selection, as well as applications of the threshold autoregressive model and its generalizations in finance.

KeYWords AND PHRASEs: Asymmetry, Heteroskedasticity, MCMC, Markov switching, Smooth transition, Nonlinearity, Volatility models.

\section{INTRODUCTION}

Nonlinear time series modelling drew much attention in the 1970's, during the time when many classes of models were proposed. As compared to the linear models, the nonlinear time series models provide a much wider spectrum of possible dynamics for economic and financial time series data. The threshold autoregressive (TAR) model is proposed by Tong $(1978,1983)$ and Tong and Lim (1980) for describing periodic time series. This model captures the dynamic behavior of time series by switching the regimes. The features of this class of models include limit cycles, amplitude dependent frequencies and jump phenomena which linear models fail to capture. The TAR model plays an important role in nonlinear time series modelling. The new era of nonlinear time series analysis, following the proposal of the TAR model, offers us very exciting possibilities. A general form of Tong's TAR model is given as follows:

$$
y_{t}=\phi_{0}^{\left(J_{t}\right)}+\sum_{i=1}^{p} \phi_{i}^{\left(J_{t}\right)} y_{t-i}+\theta^{\left(J_{t}\right)} a_{t}
$$

where $a_{t}$ s are i.i.d. $D\left(0, \sigma^{2}\right)$ and $\left\{J_{t}\right\}$ are indicator random variables, taking integer values in $\{1, \ldots, J\}$. Typical threshold modelling assumes that the value of $J_{t}$ is determined by

*Corresponding author. a threshold variable $z_{t} .\left\{J_{t}\right\}$ can be a Markov chain driven TAR model. The Markov chain can be either observable or hidden. (see Tong and Lim 1980, p. 285, Tong 1983, p. 62 , and Tyssedal and Tjøstheim 1988). When $\left\{J_{t}\right\}$ is hidden, (1) is also called Markov switching models (Hamilton 1989). The indicator time series acts as a switching mechanism. Note that the TAR model can be easily extended to a threshold autoregressive moving average model (TARMA model for short) by replacing $\theta^{\left(J_{t}\right)} a_{t}$ with $\sum_{j=0}^{q} \theta_{j}^{\left(J_{t}\right)} a_{t-j}$. Further extension to include some exogenous time series is straightforward and called TARMAX.

It is well known that financial market volatility changes over time and often exhibits the volatility clustering property: large changes in prices tend to cluster together, resulting in the persistence of the amplitudes of price changes. A common way to capture this phenomenon is that proposed by Engle (1982) who introduces the autoregressive conditional heteroscedastic (ARCH) model for modelling timevarying conditional variance of financial returns. Bollerslev (1986) proposes an extension to a generalized ARCH (GARCH) model which is a widely accepted model to describe the time series properties of financial market returns. However, symmetric ARCH and GARCH formulations are not well suited for capturing an asymmetric response of volatility. This phenomenon is discovered by Black (1976) and subsequently confirmed by Nelson (1991) and Glosten, Jagannathan and Runkle (1993), among others. Numerous improvements have been made to the GARCH model. For example, Chan (2009) provides a useful tool for researchers and students interested in the theory and practice of nonlinear time series analysis, while Li (2009) examines threshold approaches in volatility modelling. The idea of nonlinearity is also extended to the threshold unit-root test and the discontinuous adjustment to a long-run equilibrium (threshold co-integration), e.g. Kapetanios, Shin, and Snell (2003).

A number of books and a vast number of research papers have been published in diverse areas, such as ecology, econometrics, economics, finance, actuarial science and hydrology, motivated by Tong's threshold models. The goal of this paper is to give a thorough review of the vast and important development of the threshold time series model in financial applications and to provide a streamlined approach to financial time series analysis. This paper is organized as follows. We discuss representations of the threshold time series models in Section 2. Testing for the presence of 
nonlinearity is described in Section 3. For the estimation of threshold models, two major approaches, frequentist and Bayesian approaches, are discussed in Section 4. Many issues related to model selection and diagnostic checking are addressed in Section 5. Section 6 provides a review of recent applications of the threshold models in finance. Section 7 concludes.

\section{THRESHOLD MODELLING}

In this section, we describe three classes of threshold time series models, namely nonlinearity in the mean (or level), nonlinearity in the volatility, and 'double' threshold dynamics (in mean and volatility). Franses and van Dijk (2000) give an excellent review on model specifications and basic properties of the first two classes. In this survey, we also include recent developments in multivariate threshold modelling with a particular focus on financial applications.

\subsection{Nonlinearity in the mean}

The self-exciting TAR (SETAR) model defined in Tong and Lim (1980) is characterized by the lagged endogenous variable, $y_{t-d}$. The threshold variable in (1) can also be determined by an exogenous time series $X_{t}$, as in Chen (1998). Other choices of $z_{t}$ include linear combinations of the lag variables or exogenous variables (Chen, Chiang and So 2003, Chen and So 2006 and Gerlach, Chen, Lin, and Huang 2006) and nonlinear combinations (Chen 1995, Wu and Chen 2007). A special case of the TAR model is the switching autoregressive model, as originally proposed in Tong and Lim (1980), subsequently formalized by Hamilton (1989), and used by McCulloch and Tsay (1994). This model uses a random latent (unobservable) indicator as the threshold variable. The mixture autoregressive models of Wong and Li (2000a) and Wong, Chan and Kam (2009) also fall into this category.

A range of variants on the threshold nonlinear model structure have also been proposed, including the threshold moving-average (TMA) (Tong 1990, De Gooijer 1998, Ling, Tong and $\mathrm{Li}$ 2007) and threshold autoregressive movingaverage models (TARMA) (Tong 1990, Ling 1999, Amendola, Niglio and Vitale 2006). These extensions have led to applied and theoretical works in this model class. Even though the TMA model is quite well studied in the statistics literature, there has been less focus on TMA/TARMA in financial applications. This is likely due to the well-known difficulty of including moving-average terms in the model. Comparing nonlinear models with linear ARMA models where there are well-documented stationarity and invertibility conditions, similar statistical properties in the threshold class of models are far more complicated. For the threshold time series models, such as TAR, TMA and TARMA models, the stationary and invertible conditions in previous research are summarized in Table 1 . The previous studies are restricted to the AR process (or MA process) with order one in the threshold model, and there are only sufficient conditions for general orders. It remains difficult to find necessary and sufficient conditions for stationarity and invertibility in TARMA models.

In addition, it is possible to have an indicator function, $J_{t}$, to present the threshold and to enable a smooth and continuous transition for regime switching, an idea first proposed by Bacon and Watts (1971) and introduced to nonlinear time series models by Chan and Tong (1986). This smooth transition method later gains popularity (Granger and Teräsvirta 1993, Teräsvirta 1994, Teräsvirta 1998). van Dijk, Teräsvirta and Franses (2002) give a comprehensive review of the smooth transition autoregressive (STAR) model and several of its variants, such as basic STAR models (logistic or exponential STAR models), multiple regime STAR models and vector STAR models. The basic STAR model can be specified as follows:

$$
\begin{aligned}
y_{t}= & \left(1-G\left(y_{t-d} ; \gamma, c\right)\right)\left[\phi_{0}^{(1)}+\sum_{i=1}^{p_{1}} \phi_{i}^{(1)} y_{t-i}\right] \\
& +G\left(y_{t-d} ; \gamma, c\right)\left[\phi_{0}^{(2)}+\sum_{i=1}^{p_{2}} \phi_{i}^{(2)} y_{t-i}\right]+a_{t}
\end{aligned}
$$

where the transition function $G\left(y_{t-d} ; \gamma, c\right)$ is usually assumed to be a logistic, exponential or a cumulative distribution function. The observation $y_{t}$ is determined as a weighted average of two AR processes. The parameter $c$ is interpreted as the threshold and $\gamma$ is the parameter of smoothness for the smooth transition. A logistic function specification for $G($.$) is perhaps the most common choice in$ the literature.

\subsection{Nonlinearity in the volatility}

Volatility, usually defined as the standard deviation of financial returns, is a common measure of risk in financial time series. It is also a key input to many investment decisions such as the calculation of derivative prices, portfolio selection and risk management. The family of GARCH models is one of the most popular volatility models among practitioners. Poon and Granger (2003) note several salient features in financial time series and well document the measurement of volatility and volatility forecasts. These include fattailed distributions of risky asset returns, volatility clustering, asymmetry and mean reversion. Asymmetry in volatility is first discussed by Black (1976) and later by Christie (1982), from which a major cause of the asymmetry is recognized as the leverage effect. It has now become standard to use asymmetric (often nonlinear) GARCH-type models to describe the characteristics of financial time series. Li (2009) gives an extensive survey on the threshold approaches to model volatility changes.

The GJR-GARCH model given below (named after Glosten et al. 1993) can be interpreted as a special case 
Table 1. Stationary and invertible conditions for various TAR, TMA and TARMA models

\begin{tabular}{|c|c|c|}
\hline & Considered Model & Conditions \\
\hline $\begin{array}{l}\text { Petruccelli and } \\
\text { Woolford (1984) }\end{array}$ & Two-regime $\operatorname{TAR}(1), d=1$ & $\begin{array}{l}\text { Necessary and sufficient condition } \\
\text { for the ergodicity: } \\
\phi_{1}^{(1)}<1, \phi_{1}^{(2)}<1 \text { and } \phi_{1}^{(1)} \phi_{1}^{(2)}<1\end{array}$ \\
\hline Chen and Tsay (1991) & $\begin{array}{l}\text { Two-regime TAR }(1) \text {, } \\
\text { with general } d\end{array}$ & $\begin{array}{l}\text { Necessary and sufficient condition } \\
\text { for the ergodicity: } \\
\phi_{1}^{(1)}<1, \phi_{1}^{(2)}<1, \phi_{1}^{(1)} \phi_{1}^{(2)}<1, \\
\phi_{1}^{(1)^{s(d)}} \phi_{1}^{(2)^{t(d)}}<1 \\
\text { and } \phi_{1}^{(1)^{t(d)}} \phi_{1}^{(2)^{s(d)}}<1 \\
\text { where } s(d) \text { and } t(d) \text { are nonnegative } \\
\text { integers depending on } d\end{array}$ \\
\hline Tong (1983) & $k$-regime TAR $(1)$ & $\begin{array}{l}\text { Sufficient condition for the existence } \\
\text { of stationarity: } \\
\left|\phi_{1}^{(1)}\right|<1 \text { and }\left|\phi_{1}^{(k)}\right|<1\end{array}$ \\
\hline $\begin{array}{l}\text { Chan, Petruccelli, Tong } \\
\text { and Woolford (1985) }\end{array}$ & $k$-regime TAR $(1)$ & $\begin{array}{l}\text { Sufficient condition for stationarity } \\
\text { and ergodicity: } \\
\phi_{1}^{(1)}<1, \phi_{1}^{(k)}<1 \text { and } \phi_{1}^{(1)} \phi_{1}^{(k)}<1\end{array}$ \\
\hline Chan and Tong (1985) & $k$-regime $\operatorname{TAR}(p)$ & $\begin{array}{l}\text { Sufficient condition for stationarity } \\
\text { and ergodicity: } \\
\max _{j} \sum_{i=1}^{p}\left|\phi_{i}^{(j)}\right|<1, \quad j=1, \ldots, k\end{array}$ \\
\hline $\begin{array}{l}\text { Brockwell, Liu and } \\
\text { Tweedie (1992) }\end{array}$ & $\begin{array}{l}k \text {-regime TARMA }(p, q), \\
\text { here MA is assumed linear }\end{array}$ & $\begin{array}{l}\text { Sufficient condition for stationarity } \\
\text { and ergodicity: } \\
\rho\left(\max _{j}\left\{|\bar{\Phi}|^{(j)}\right\}\right)<1 \\
\text { where } \bar{\Phi} \text { is a specified matrix } \\
\text { which contains ARMA coefficients }\end{array}$ \\
\hline$\overline{\text { Ling (1999) }}$ & $\begin{array}{l}\text { General } k \text {-regime } \\
\operatorname{TARMA}(p, q)\end{array}$ & $\begin{array}{l}\text { Strictly stationary condition: } \\
\sum_{i=1}^{p} \max _{j}\left|\phi_{i}^{(j)}\right|<1, j=1, \ldots, k\end{array}$ \\
\hline Ling and Tong (2005) & Two-regime TMA(1) & $\begin{array}{l}\text { Invertible condition: } \\
\left|\theta_{1}^{(1)}\right|<1 \text { and }\left|\theta_{1}^{(2)}\right|<1\end{array}$ \\
\hline \multirow[t]{2}{*}{ Ling, Tong and Li (2007) } & Two-regime TMA(1) & $\begin{array}{l}\text { A weaker invertible condition: } \\
\left|\theta_{1}^{(1)}\right|^{1-F_{y}(r)}\left|\theta_{1}^{(2)}\right|{ }^{F_{y}(r)}<1 \\
\text { where } F_{y} \text { is the distribution function } \\
\text { of } \boldsymbol{y} \text { and } r \text { is the threshold value }\end{array}$ \\
\hline & $k$-regime $\mathrm{TMA}(1)$ & $\begin{array}{l}\text { Invertible condition: } \\
\prod_{j=1}^{k}\left|\theta_{1}^{(j)}\right|^{F_{y}\left(r_{j}\right)-F_{y}\left(r_{j-1}\right)}<1 \\
\text { where } r_{j} \text { is the threshold value of } \\
\text { regime } j\end{array}$ \\
\hline
\end{tabular}

of threshold model.

$$
\begin{gathered}
y_{t}=a_{t}, \quad a_{t}=\sqrt{h_{t}} \epsilon_{t}, \quad \epsilon_{t} \sim D(0,1), \\
h_{t}=\alpha_{0}+\sum_{j=1}^{p} \alpha_{j} a_{t-j}^{2} \\
\quad+\sum_{j=1}^{p} \gamma_{j} S_{t-j}^{-} a_{t-j}^{2}+\sum_{k=1}^{q} \beta_{k} h_{t-k}, \\
\text { where } S_{t-j}^{-}= \begin{cases}1 & \text { if } a_{t-j} \leq 0, \\
0 & \text { if } a_{t-j}>0,\end{cases}
\end{gathered}
$$

$D(0,1)$ is a distribution with mean 0 and variance 1 . Glosten et al. (1993) use dummy variables for negative shocks in the volatility equation and find evidence that local negative market news causes increased market volatility. This finding is confirmed in studies by Koutmos (1999) and Nam, Pyun and Avard (2001), etc. The GJR-GARCH model is popular in the finance literature, as it explains the asymmetric volatility phenomenon through its simplicity and clear interpretation of its asymmetric volatility parameter. Zakoian (1994) and Rabemananjara and Zakoian (1993) are two early works in modelling volatility equation using threshold type models. The former extends $\mathrm{ARCH}$ to a piecewise linear function of the squared past return innovations. Rabemananjara and Zakoian (1993) study a more relaxable version of the threshold heteroscedastic model in Zakoian (1994).

In addition to the threshold type models, Nelson (1991) introduces an exponential GARCH (EGARCH) model to allow for asymmetric effects of market news. Hagerud (1996) and González-Rivera (1998) independently adopt the idea of smooth transition to allow for a more gradual change in the squared past shocks. Anderson, Nam and Vahid (1999) modify a volatility switching GARCH model by allowing the transition from one regime to the other regime to be smooth. 
Table 2. The conditions of stationarity and positiveness of volatility for nonlinear heteroscedastic models

\begin{tabular}{|c|c|c|}
\hline & Considered Model & Conditions \\
\hline Glosten, Jagannathan & GJR-GARCH $(1,1)$ & $\alpha_{0}>0, \beta_{1}>0$ \\
\hline and Runkle (1993) & $\begin{array}{l}h_{t}=\alpha_{0}+\left(\alpha_{1}+\gamma I_{t-1}\right) a_{t-1}^{2}+\beta_{1} h_{t-1} \\
\text { where } I_{t-1}=I_{\left(a_{t-1}>0\right)}\end{array}$ & $\begin{array}{l}\alpha_{1}>0, \alpha_{1}+\gamma \geq 0 \\
\text { and } \alpha_{1}+\beta_{1}+0.5 \gamma<1\end{array}$ \\
\hline Hagerud (1996) & $\begin{array}{l}\text { ST-GARCH }\left(p_{j}, q_{j}\right) \text { with } 2 \text { regimes } \\
h_{t}=\alpha_{0}+\sum_{i=1}^{p}\left(\alpha_{1 i}+\alpha_{2 i} F\left(a_{t-i}\right)\right) a_{t-i}^{2} \\
+\sum_{k=1}^{q} \beta_{k} h_{t-k} \\
\text { where } F(.) \text { is an exponential transition }\end{array}$ & $\begin{array}{l}\alpha_{0}>0, \alpha_{1 i}, \beta_{i} \geq 0 \\
\alpha_{1 i}+\alpha_{2 i} \geq 0 \\
\text { and } \sum_{i}\left(\alpha_{1 i}+\alpha_{2 i}\right)+\sum_{k} \beta_{k}<1\end{array}$ \\
\hline Chen and So (2006) & DTGARCH in Eq. (4) & $\begin{array}{l}\alpha_{0}^{(j)}>0 ; \alpha_{i}^{(j)}, \beta_{l}^{(j)}>0 ; j=1, \ldots, g \\
\sum_{i} \alpha_{i}^{(j)}+\sum_{k} \beta_{k}^{(j)}<1\end{array}$ \\
\hline $\begin{array}{l}\text { Chen, So } \\
\text { and Gerlach (2005) }\end{array}$ & $\begin{array}{l}\text { Threshold GJR-GARCH }(1,1) \text { with } 2 \text { regimes } \\
h_{t}=\alpha_{0}^{(j)}+\left(\alpha_{1}^{(j)}+\gamma^{(j)} I_{t-1}\right) a_{t-1}^{2}+\beta_{1}^{(j)} h_{t-1} \\
\text { where } I_{t-1}=I_{\left(a_{t-1}>0\right)}\end{array}$ & $\begin{array}{l}\alpha_{0}^{(j)}>0, \alpha_{1}^{(j)}, \beta_{1}^{(j)} \geq 0 \\
\alpha_{1}^{(j)}+\gamma^{(j)} \geq 0 \\
\text { and } \alpha_{1}^{(j)}+\beta_{1}^{(j)}+0.5 \gamma^{(j)}<1\end{array}$ \\
\hline $\begin{array}{l}\text { Chen, Gerlach } \\
\text { and Lin (2008) }\end{array}$ & $\begin{array}{l}\operatorname{TARR}\left(p_{j}, q_{j}\right) \text { with } k \text { regimes } \\
\lambda_{t}=\alpha_{0}^{(j)}+\sum_{i=1}^{p_{j}} \alpha_{i}^{(j)} R_{t-i}+\sum_{k=1}^{q_{j}} \beta_{k}^{(j)} \lambda_{t-k} \\
\text { where } R_{t} \text { is the intra-day log price range }\end{array}$ & $\begin{array}{l}\alpha_{0}^{(j)}>0, \alpha_{i}^{(j)}, \beta_{i}^{(j)} \geq 0 \\
\text { and } \sum_{i} \alpha_{i}^{(j)}+\sum_{k} \beta_{k}^{(j)}<1\end{array}$ \\
\hline $\begin{array}{l}\text { Gerlach and } \\
\text { Chen (2008) }\end{array}$ & ST-GARCH in Eq. (13) & $\begin{array}{l}\alpha_{0}^{(1)}>0, \alpha_{i}^{(1)}, \beta_{i}^{(1)} \geq 0 \\
\sum_{i} \alpha_{i}^{(1)}+\alpha_{i}^{(2)} \geq 0, \sum_{i} \beta_{i}^{(1)}+\beta_{i}^{(2)} \geq 0, \text { and } \\
\sum_{i}\left(\alpha_{i}^{(1)}+0.5 \alpha_{i}^{(2)}\right)+\sum_{j}\left(\beta_{j}^{(1)}+0.5 \beta_{j}^{(2)}\right)<1 \\
\alpha_{0}^{(1)}<b_{1}, \beta_{i}^{(1)}<b_{2}, \sum_{i} \alpha_{i}^{(1)}+\sum_{j} \beta_{j}^{(1)}<b_{3} \\
b_{2}, b_{3} \geq 1 \text { to allow explosive behavior }\end{array}$ \\
\hline $\begin{array}{l}\text { Chen, Gerlach } \\
\text { and Lin (2010) }\end{array}$ & DTGARCH in Eq. (4) & $\begin{array}{l}\alpha_{0}^{(j)}>0 ; \alpha_{i}^{(j)}, \beta_{l}^{(j)}>0 ; j=1, \ldots, g \\
\sum_{j} \operatorname{Pr}\left(R_{j}\right) \times\left(\sum_{i} \alpha_{i}^{(j)}+\sum_{k} \beta_{k}^{(j)}\right)<1 \\
\text { where } \operatorname{Pr}\left(R_{j}\right) \text { denotes the probability of regime } j\end{array}$ \\
\hline
\end{tabular}

The parameter restrictions to ensure stationarity and positiveness of volatility for each nonlinear model are given in Table 2. Note that the feature of DTGARCH models in Chen, Gerlach, and Lin (2010) is that the overall stationarity does not require the model to be stationary in each regime. On the contrary, the limit cycle behavior that this class of models is able to demonstrate arises from the alternation of explosive, dormant, and rising regimes. The sufficient conditions on the parameters to ensure positive variance and covariance stationary are given in the last row of Table 2.

\subsection{Nonlinearity in the mean and volatility}

Motivated by the TAR model of Tong (1978, 1990), $\mathrm{Li}$ and $\mathrm{Li}$ (1996) propose a double threshold ARCH (DTARCH), also allowing asymmetric mean responses, which is extended to the double threshold GARCH (DTGARCH) model by Brooks (2001). These models have been used to assess asymmetry via threshold nonlinearity in major market indices, daily stock returns, exchange rates and other financial variables (e.g. Koutmos 1999 and Chen et al. 2003). Chen, So and Gerlach (2005) present clear evidence that the double threshold GARCH model, with a local news threshold, outperforms the symmetric GARCH model in the G7 stock markets.

Chen and So (2006) further allow the threshold variable for regime switching to be formulated as a weighted average of important auxiliary variables. Estimation and diagnostic checks are performed using Bayesian methods. Their multiregime models can be represented as follows:

$$
\begin{aligned}
y_{t}= & \phi_{0}^{(j)}+\sum_{i=1}^{p_{j}} \phi_{i}^{(j)} y_{t-i}+\sum_{k=1}^{q_{j}} \psi_{k}^{(j)} x_{k t}+a_{t}, \\
& \quad \text { if } r_{j-1} \leq z_{t-d}<r_{j}, \\
a_{t}= & \sqrt{h_{t}} \epsilon_{t}, \quad \epsilon_{t} \sim D(0,1), \\
h_{t}= & \alpha_{0}^{(j)}+\sum_{i=1}^{d_{j}} \alpha_{i}^{(j)} a_{t-i}^{2}+\sum_{k=1}^{c_{j}} \beta_{k}^{(j)} h_{t-k},
\end{aligned}
$$

where

$$
\text { (5) } \quad z_{t}=w_{1} z_{1 t}+\cdots+w_{l} z_{l t}, \quad 0 \leq w_{i} \leq 1, \quad \sum_{i=1}^{l} w_{i}=1
$$

$j=1, \ldots, g, d$ is a positive integer. The model assumes $g$ regimes defined by the threshold values $r_{j}$ satisfying $-\infty=$ $r_{0}<r_{1}<\cdots<r_{g}=\infty$ and the $d$-period delayed threshold variable $z_{t-d}$. For identification purposes, $\phi_{p_{j}}^{(j)} \neq 0$ and the autoregressive vectors $\left(\phi_{0}^{(j)}, \ldots, \phi_{p_{j}}^{(j)}\right), j=1, \ldots, g$, are assumed to be different. One main advantage of the threshold heteroscedastic model in (4) and (5) is to construct the threshold variable $z_{t}$ as a linear function of $m$ auxiliary variables $z_{i t}$. In general, $z_{i t}$ can be any function of exogenous variables and $y_{t}, \ldots, y_{1}$. A likely choice is to set $z_{1 t}=y_{t}$ 
and $z_{i t}=x_{i t}$ for $i>1$ where $x_{i t}$ are exogenous variables. In this case, the information in both $y_{t}$ and exogenous variables can be used to govern the threshold switching. Chen and So (2006) use this choice of $z_{i t}$ in their empirical analysis and find that in most of the markets investigated, rather than international returns, domestic return is the dominating factor for determining the threshold.

Instead of having a two-regime structure, Chen, Gerlach and Lin $(2010,2011)$ investigate three-regime DTGARCH models which help to identify explosive, dormant and rising scenarios to capture the mean and volatility asymmetries. Their proposed method allows simultaneous inference for all unknown parameters, including the problems of estimating threshold limits and delay lags. Extending from the use of step functions in the threshold characterization, Gerlach and Chen (2008) propose a double smooth transition GARCH (DST-GARCH) model and design an adaptive Markov chain Monte Carlo scheme to investigate the estimation and forecasting performance of the models. A DSTGARCH model with smooth transition functions is given in the Appendix. Chen, Gerlach, Choy, and Lin (2010) consider a family of threshold nonlinear GARCH models that allows exponential smooth transitions between regimes, capturing size asymmetry via an exponential smooth transition function. In addition, Audrino and Bühlmann (2001) propose a tree-based GARCH model to incorporate threshold volatility features.

The Stochastic Volatility (SV) model proposed by Taylor (1986) provides an alternative to GARCH models in accounting for the time-varying and persistent volatility as well as for the leptokurtosis in financial series. The SV model has the advantage that it includes an additional innovation term, to provide a more flexible description of time-varying volatility. In addition to the threshold in ARCH or GARCH, So, Li, and Lam (2002) assume threshold nonlinearity under stochastic volatility models. To capture mean and volatility asymmetries, the threshold SV model in So, Li, and Lam (2002) is divided into two distinct regimes in response to lagone bad news $\left(y_{t-1}<0\right)$ and lag-one good news $\left(y_{t-1} \geq 0\right)$. A generalization of the THSV model is introduced by Chen, Liu and So (2008), for which the specifications are given in the Appendix. Chen et al. (2008) extend the So, Li, and Lam (2002) model to have the error innovations following the standardized t-distribution. They also include exogenous variables in the mean equation for detecting possible asymmetries in the spillover effect from other financial markets. Instead of using an observed time series to define the threshold structure, So, Lam and Li (1998) study a Markov switching SV model where the threshold dynamic is governed by a Markov process independent of the return time series. Generalizing from Wong and $\mathrm{Li}$ (2000a), Wong and $\mathrm{Li}$ (2001) develop a mixture autoregressive ARCH model. Extending from daily return applications to possibly high-frequency return applications, McAleer and Medeiros (2008) propose a smooth transition heterogenous autoregressive model. The methodology of McAleer and Medeiros (2008) can model the realized volatility that is computed from intraday return data.

\subsection{Multivariate threshold models}

In the first two decades of threshold modelling development, most studies center on univariate analysis. Due to the high-dimensional nature of economic and financial data, univariate analysis may not be adequate to tackle a wide range of business problems. Understanding and predicting the temporal dependence in the second order moments of asset returns is important for many issues in financial econometrics. It is now widely accepted that financial volatilities move together over time across assets and markets. Recognizing this feature through a multivariate modelling framework should lead to more relevant empirical models than working with separate univariate models. To the best of our knowledge, Tsay (1998) is the first to perform a comprehensive investigation on multivariate threshold modelling. His motivation example draws from the arbitrage theory for spot and future prices. In his paper, Tsay proposes the use of arranged autoregression and develops a multivariate test for the threshold dynamic.

The study of the time-varying conditional covariance dates back to Bollerslev, Engle and Wooldridge (1988) who suggest a basic multivariate GARCH (MGARCH) model. De Goeij and Marquering (2004) extend the univariate GJR model to a multivariate GJR structure to allow for asymmetries in conditional variances and covariances. Using weekly U.S. stock and bond market data, their results indicate that variances and covariances respond asymmetrically to return shocks. Pelletier (2006) introduces a regime switching model of constant correlations within each regime. The timevarying correlation model provides more realistic empirical results than the dynamic conditional correlation model proposed by Engle (2002). McAleer, Hoti and Chan (2009) propose an asymmetric MGARCH model where some parameters depend on the sign of the innovations. Caporin and McAleer (2006) develop the dynamic asymmetric GARCH (or DAGARCH) model to generalize asymmetric GARCH models with multiple thresholds and to make the asymmetric effect time dependent. They provide stationarity conditions for the DAGARCH model and derive the news impact curve surface to analyze the multivariate asymmetric effect. The recent works of Tse and Tsui (2002) and Engle (2002) describe a new class of MGARCH models that explicitly capture the time-varying correlation matrix instead of assuming a constant-correlation formulation for the conditional covariance equation. Kwan, $\mathrm{Li}$ and $\mathrm{Ng}$ (2010) extend the Tse and Tsui (2002) model to allow the correlation equation to have threshold structure. So and Yip (2010) work along the lines of Kwan et al. (2010) to propose an aggregate threshold dynamic. So and Yip (2011) develop a correlation clustering model which allows correlation dynamic 
to follow different regimes. Audrino and Trojani (2006) develop a tree-based MGARCH model for explaining correlation asymmetry.

In addition to the GARCH approach, threshold volatility models are also built under the stochastic volatility (SV) framework. Asai and McAleer (2006) propose two types of asymmetric multivariate stochastic volatility models, namely, the SV with leverage (SV-L) model and the SV with leverage and size effect (SV-LSE) model. They adopt the Monte Carlo likelihood (MCL) estimation for their multivariate SV-L and SV-LSE models. The empirical results show that the multivariate SV-LSE model fits the data more accurately than the multivariate SV-L model. Generalizing the multivariate SV models in Harvey, Ruiz and Shephard (1994) and So, Li and Lam (1997), So and Choi (2008) develop a multivariate threshold SV model and Bayesian analysis for the model. Through the model, smoothed conditional correlations can also be calculated. To allow highdimensional implementation, So and Choi (2009) include a factor structure in the So and Choi (2008) model as in Chib, Nardari and Shephard (2006). By applying the factor threshold SV model of So and Choi (2009) to 20 time series, a two-factor model produces a reasonable fit as a three-factor model. News impact analysis is conducted to understand the effect of positive and negative news on the changes in volatility.

\section{NONLINEARITY TESTS IN THRESHOLD MODELS}

\subsection{Testing nonlinearity in mean}

In nonlinear time series modelling, it is instrumental to perform tests of nonlinearity to examine the statistical adequacy of linear models and to detect possible nonlinearity features in time series. A general representation of nonlinear stationary time series model is referred to as the Volterra expansion. Keenan (1985) constructs an analogue of Tukey's (1949) one degree of freedom nonadditivity test for linearity with the second-order Volterra expansion as an alternative. Tsay (1986) modifies the Keenan (1985) test and retains its simplicity to obtain a more powerful test for nonlinearity. Petruccelli and Davies (1986) propose a portmanteau test to detect self-exciting threshold autoregressive-type nonlinearity in time series data. The test is based on cumulative sums of standardized residuals from autoregressive fits to the data. Incorporating the nonlinearity tests of Keenan (1985), Tsay (1986), and Petruccelli and Davies (1986), Tsay (1989) proposes a portmanteau test based on arranged autoregression and predictive residuals. The test statistic is a usual $F$ statistic which can be implemented by sorting routine and linear regression. For multivariate time series modelling, Tasy (1998) generalizes the test of Tsay (1989) for detecting threshold nonlinearity in vector time series. Based on the likelihood ratio idea, Chan (1990) developes a test for threshold autoregressive processes. Wong and Li (1997) extend the Chan (1990) test to incorporate heteroscedasticity. Wong and $\mathrm{Li}$ (2000b) further generalize the results of Wong and Li (1997) to also allow threshold structure in the mean.

\subsection{Testing nonlinearity in volatility}

Engle and Ng (1993) propose a set of tests for asymmetry in volatility, known as sign and size bias tests. The Engle and $\mathrm{Ng}$ tests are useful for determining whether an asymmetric model is required for a given series, or whether the symmetric GARCH model can be deemed adequate. Apart from the tests based on residuals, there are Bayesian approaches via MCMC methods in the literature. In Chen, Gerlach and Tai (2008), a nonlinearity test for mean and volatility for heteroscedastic models is proposed by using posterior credible intervals on model parameters. The test proceeds by fitting the DTGARCH model to the data using MCMC methods. Then, posterior credible intervals of the differences in mean and volatility parameters are obtained from MCMC samples for the testing. The testing problem can also be referred to as a Bayesian model selection problem. So, Chen and Chen (2005) test the significance of the threshold nonlinearity by comparing a GARCH and its threshold GARCH counterpart. The reversible-jump MCMC (RJMCMC) method of Green (1995) is used to compute the posterior probabilities of GARCH and threshold GARCH models. Then, a better model can be selected with a higher posterior probability, and we can declare significant volatility asymmetry effect if the threshold GARCH model is preferred. One advantageous feature of the Bayesian test is that it can simultaneously account for uncertainty in the unobserved threshold value and the time delay lag parameter while performing the nonlinearity test. In fact, there is little work focusing on procedures for model choice or comparison among competing asymmetric volatility models. The same is true for allowance of uncertainty about threshold values (usually set to 0 ) and delay lags (set to 1). Following So et al. (2005), Chen et al. (2005) adopt the RJMCMC idea to test for threshold nonlinearity in a time series with GARCH volatility dynamics in G-7 stock markets. Posterior evidence favoring the threshold GARCH model indicates threshold nonlinearity with asymmetric behaviors in the mean and volatility. In addition, Chen, Gerlach and So (2006) design a RJMCMC procedure, allowing jumps between potentially nonnested models of different dimensions to estimate posterior probabilities between pairs of competing nonlinear models. Adopting a direct estimation method proposed by Congdon $(2006,2007)$ to estimate posterior probabilities, Chen, Gerlach and So (2008) compare seven heteroscedastic models for three daily Asian market returns. The details of direct estimation method of Congdon $(2006,2007)$ will be discussed in a later section.

\section{STATISTICAL INFERENCE}

Studying financial data is one of the most active areas in time series research over the last three decades. There are 
a large (and still growing) number of papers using complex time series models. For the estimation of threshold models, frequentist approach and Bayesian Monte Carlo simulation are the two major methods in the literature. Thus, the key objectives are to review threshold time series model in finance and economics over frequentist and Bayesian approaches.

\subsection{Frequentist approach}

Estimation of parameters in the threshold models is complex. Quantities to be determined include the number of regimes, the threshold variable, the delay parameter, the model order in each regime, the threshold values, and the coefficients in each regime. One challenge in threshold modelling is to determine the delay parameter and the threshold value. In the previous literature, a two-stage approach is commonly adopted (see Tong and Lim 1980, Ghaddar and Tong 1981, Tsay 1989). The asymptotic properties of the least squares estimation for TAR are established by Chan (1993). Hansen (1997) investigate statistical inference procedures of the TAR models.

The two-stage approach specifies the delay parameter of the threshold variable and the threshold values and then uses the likelihood-based maximization to estimate the model parameters. With the two-stage approach, Tsay (1989) estimates the parameters of two-regime threshold autoregressive (TAR) models by ordinary least squares. The least squares estimators of the TAR models are consistent and converge almost surely to the true values given the threshold variables and threshold values. For the specification of the delay lag and the threshold values, Tong and Lim (1980) and Ghaddar and Tong (1981) use the Akaike information criterion (Akaike 1974) to select the delay lag after choosing all of the other parameters. Tsay (1989) proposes a testing procedure to select the delay lag and uses scatter plots to identify the thresholds. Liu (2009) addresses the issue of TAR model estimation by using the weighted estimation method. In the mixture models of Wong and Li (2000a, 2001) where the regimes are governed by latent variables, the EM algorithm is adopted.

\subsection{Bayesian approach for two-regime models}

Practitioners are increasingly turning to Bayesian methods for the analysis of complicated heteroscedastic models. This move seems due to the advent of inexpensive high speed computers and the development of stochastic integration methodology, especially Markov Chain Monte Carlo (MCMC) approaches. MCMC is a computationally intensive simulation method for numerical integration developed in the 1980s, making it possible to tackle more complex, realistic models and problems.

This subsection discusses how Bayesian methods can be used to cope with challenges that arise in dynamic heteroscedastic and SV models in finance. Bayesian approach via MCMC methods is successfully applied to the family of TAR models (e.g. Chen and Lee 1995, Chen 1998, So and Chen 2003). The MCMC idea is a sequential sampling scheme which enables us to decompose high-dimensional parameter estimation to sampling of low dimensional blocks and thus makes high-dimensional estimation feasible. As such, estimation of all threshold parameters, including the delay and threshold values, can be done at the same time. Unlike two-stage frequentist methods, the Bayesian approach can take into account estimation errors of the delay and threshold values when estimating other parameters. Chen and Lee (1995) perform simultaneous inference for all parameters, including the delay lag of threshold variable and the threshold value, of TAR models by Bayesian approach. The samples of parameter estimates are generated from the joint posterior distribution of all parameters via MCMC methods. Applications of the Bayesian approach can also be found in Chen (1998) for generalized TAR models, in Chen et al. (2003) for investigation of the asymmetric reaction of US stock market with DTGARCH models, in So and Chen (2003) for subset selection of TAR models, and in Chen, Lin, Liu, and Gerlach (2008) for the estimation of parsimonious TAR models.

One advantage of the Bayesian approach is to incorporate parameter restrictions, such as those from stationarity and positivity conditions, to be incorporated into the prior distribution. By specifying priors with suitable domain, the posterior sample of parameters automatically satisfies any necessary constraint. Furthermore, we can allow the threshold variable to depend on unknown parameters as in Chen and So (2006). Advances in Bayesian computations let the researcher efficiently deal with numerical complications that arise in models with latent state variables, such as regimeswitching GARCH models (Chen, So, and Lin 2009 and Bauwens, Preminger and Rombouts 2010).

\subsection{Multiple-regime threshold models}

Many authors contributing to threshold models find significant nonlinearity and/or asymmetry in the volatility, and the mean or level, of financial market and asset return data. Most of these studies focus only on two-regime models. There are some exceptions, for example Brooks and Garrett (2002) consider a three-regime TAR model to explain the (mean) dynamics of spot and futures markets, but the delay lag is subjectively fixed at one and no formal model comparison is considered. Boeroa and Marrocu (2004) evaluate the forecasting performance of a two-regime and a threeregime SETAR model, using a three-stage procedure to estimate the parameters. In two and three-stage estimation approaches uncertainty about the threshold lag and threshold value is not properly included in the analysis, which is less than optimal. One issue is that if the chosen threshold or delay parameters are changed, the estimation and forecasts of the SETAR model can be substantially different. Chen, Gerlach, and Lin (2010) propose an efficient way 
to make inference and approximate model comparisons for a general multiple-regime nonlinear DT-GARCH specification, where the number of regimes can be greater than two. This paper allows for an explosive volatility regime. So, Lam, and $\mathrm{Li}$ (1998) also consider a three regime Markov-switching stochastic volatility model, but focus on the intercept change between regimes.

Capital asset pricing model (CAPM) has become an essential tool in finance for assessing the cost of capital, risk management, portfolio diversification and other financial assets. Banz (1981) and Fama and French (1992) argue that the simple constant beta is not sufficient on CAPM. Ghysels (1998) shows that continuously time-varying models for market beta do not approximate the dynamics well, and speculates that betas actually change much more slowly and discretely than implied. Chen, Gerlach, and Lin (2011) draw on Bollerslev's GARCH modelling strategy to formulate a multiple-regime CAPM with time-varying risk and expected returns. Three clear regimes are found in average returns: bear markets induce assets to collapse, bull markets induce assets to rise, and stable markets induce fairly dormant returns. These findings can have important implications for risk assessment, asset allocation, portfolio selection, and hedging decisions.

The problem of estimating three (or higher) regime SETAR and GARCH models is a challenge. Specifically, the likelihood function is non-differentiable in terms of the threshold values and is often multi-modal. Such problems are magnified as the number of regimes increases. Further, quasi-likelihood approaches can also be sub-optimal under the usual parameter constraints applied to GARCH models. Bayesian approaches, based on MCMC methods, allow simultaneous inference for all unknown parameters, while parameter constraints simply and properly form part of the prior distribution and problems with estimating threshold limits and delay lags disappear. Chen, Lin, and Yu (2011) consider time-varying market betas in CAPM by a smooth transition regime switching CAPM with heteroscedasticity, which provides flexible nonlinear representation of market betas as well as flexible asymmetry and clustering in volatility. This paper employs the quantile regression to investigate the nonlinear behavior in the market betas and volatility under various market conditions represented by different quantile levels. Parameter estimation is done by a Bayesian approach.

\section{ASSESSING THRESHOLD MODELS}

\subsection{Model selection}

An important task for financial modelling is to decide the best model from a group of candidate models. Traditionally, information criteria, such as Akaike's (AIC) and the Bayesian (BIC), are used to identify the 'best' model, by comparing all possible models. However, such methods are problematic with a large number of possible models and favoring of over-fitted models. Thus, researchers seek Bayesian methods for the model selection of a large number of candidate models. Bayes factors (Kass and Raftery 1995) are viewed as the correct way to carry out Bayesian model comparisons. Chen et al. (2008) review and investigate recently developed techniques for Bayesian estimation and model selection applied to a large group of modern asymmetric heteroscedastic models. These include the GJR-GARCH, threshold autoregression with GARCH errors, threshold GARCH, and double threshold heteroscedastic model with auxiliary threshold variables. In Bayesian model hypothesis testing, the decision between two models, $M_{i}$ versus $M_{j}$, is made by the posterior odds ratio; i.e., $M_{i}$ is chosen if $P O R_{i j}>1$.

$$
P O R_{i j}=\frac{p\left(M_{i} \mid \boldsymbol{y}^{1, n}\right)}{p\left(M_{j} \mid \boldsymbol{y}^{1, n}\right)}=\frac{p\left(\boldsymbol{y}^{1, n} \mid M_{i}\right)}{p\left(\boldsymbol{y}^{1, n} \mid M_{j}\right)} \frac{\operatorname{Pr}\left(M_{i}\right)}{\operatorname{Pr}\left(M_{j}\right)},
$$

where $\boldsymbol{y}^{1, n}$ is the observation vector; $\operatorname{Pr}\left(M_{i}\right)$ is the prior probability of model $M_{i}$ and $p\left(\boldsymbol{y}^{1, n} \mid M_{i}\right)$ is the marginal (or integrated) likelihood. A Bayes factor, $B_{i j}$, defined in Kass and Raftery (1995), is the ratio of posterior odds of $M_{i}$ to its prior odds. Thus, without any prior information on model choice, the prior odds ratio $=1$ and the $P O R_{i j}$ then becomes the Bayes factor.

$$
\begin{aligned}
& B_{i j}=\frac{p\left(\boldsymbol{y}^{1, n} \mid M_{i}\right)}{p\left(\boldsymbol{y}^{1, n} \mid M_{j}\right)}, \\
& \quad \text { where } p\left(\boldsymbol{y}^{1, n} \mid M_{i}\right)=\int p\left(\boldsymbol{y}^{1, n} \mid \boldsymbol{\theta}_{i}, M_{i}\right) p\left(\boldsymbol{\theta}_{i} \mid M_{i}\right) d \boldsymbol{\theta}_{i},
\end{aligned}
$$

and $\boldsymbol{\theta}_{i}$ is the vector of parameters in model $M_{i}$. Due to the challenge of calculating the integration in the marginal likelihood, the formal model comparison via Bayes factors remains difficult. Multidimensional integration over the parameter space obstructs the implementation of model comparisons. Several methodologies focusing on Bayesian model comparison methods are discussed below. These are the Monte Carlo approaches, avoiding the calculation of marginal likelihoods explicitly, for estimating the marginal likelihoods or selecting models.

Importance Sampling: Geweke (1995) and Gerlach, Carter and Kohn (1999) propose the importance sampling MCMC methods to estimate the marginal likelihood for each competing model. By running separate MCMC sampling schemes with sequential increase of the sample size $k$, an estimate of the marginal likelihood can be evaluated by

$$
\begin{aligned}
& \hat{p}\left(\boldsymbol{y}^{1, n} \mid M_{j}\right) \\
& \quad=\hat{p}\left(y_{1} \mid M_{j}\right) \prod_{t=2}^{n} \hat{p}\left(y_{t} \mid \boldsymbol{y}^{1, t-1}, M_{j}\right) ; \quad j=1, \ldots, m,
\end{aligned}
$$


where $m$ is the number of competing models, and, when $k \geq t$,

$$
\begin{array}{r}
\hat{p}\left(y_{t} \mid \boldsymbol{y}^{1, t-1}, M_{j}\right) \\
=\frac{\sum_{i=1}^{N} p\left(y_{t} \mid \boldsymbol{y}^{1, t-1}, \boldsymbol{\theta}_{k}^{(i)}, M_{j}\right) / p\left(\boldsymbol{y}^{t, k} \mid \boldsymbol{y}^{1, t-1}, \boldsymbol{\theta}_{k}^{(i)}, M_{j}\right)}{\sum_{i=1}^{N} 1 / p\left(\boldsymbol{y}^{t, k} \mid \boldsymbol{y}^{1, t-1}, \boldsymbol{\theta}_{k}^{(i)}, M_{j}\right)} .
\end{array}
$$

$\boldsymbol{\theta}_{k}^{(i)}$ is the $i$ th MCMC iterate sampled from the posterior $p\left(\boldsymbol{\theta}_{j} \mid \boldsymbol{y}^{1, k}, M_{j}\right)$. This method is employed successfully in Chen and So (2006) for double threshold heteroscedastic models; in Gerlach et al. (2006) for asymmetric DTGARCH models of price-volume relationships, and in So et al. (2007) for the return-volume partition of regimes.

Reversible-Jump MCMC (RJMCMC): The RJMCMC method of Green (1995) is similar to the reject-accept procedure of the Metropolis-Hastings algorithm in allowing jumps between spaces of different dimensions of competing models. The acceptance probability for a jump is computed as

$$
\begin{aligned}
\wp= & \frac{p\left(\boldsymbol{y}^{1, n} \mid \boldsymbol{\theta}_{j}, M_{j}\right) p\left(\boldsymbol{\theta}_{j} \mid M_{j}\right) \operatorname{Pr}\left(M_{j}\right) J\left(M_{i}, M_{j}\right) q_{j}\left(u_{j} \mid \boldsymbol{\theta}_{j}\right)}{p\left(\boldsymbol{y}^{1, n} \mid \boldsymbol{\theta}_{i}, M_{i}\right) p\left(\boldsymbol{\theta}_{i} \mid M_{i}\right) \operatorname{Pr}\left(M_{i}\right) J\left(M_{j}, M_{i}\right) q_{i}\left(u_{i} \mid \boldsymbol{\theta}_{i}\right)} \\
& \times\left|\frac{\partial\left(\boldsymbol{\theta}_{j}, u_{j}\right)}{\partial\left(\boldsymbol{\theta}_{i}, u_{i}\right)}\right| ;
\end{aligned}
$$

where $p\left(\boldsymbol{\theta}_{j} \mid M_{j}\right)$ is the prior distribution of the parameter, $\operatorname{Pr}\left(M_{j}\right)$ is the model prior probability and $J\left(M_{i}, M_{j}\right)$ is the probability of considering the jump from model $M_{i}$ to $M_{j}$. $q_{j}($.$) and q_{i}($.$) are the kernels of the accept-reject algorithm.$ The term of $\left|\partial\left(\boldsymbol{\theta}_{j}, u_{j}\right) / \partial\left(\boldsymbol{\theta}_{i}, u_{i}\right)\right|$ is a transformation Jacobian for the bijection between $\left(\boldsymbol{\theta}_{i}, u_{i}\right)$ and $\left(\boldsymbol{\theta}_{j}, u_{j}\right)$. Thus, the jump to model $M_{j}$ is allowed with the probability $\min \{1, \wp\}$. The model posterior probability of model $M_{j}$ can be computed by the proportion of times the MCMC sample jumps to model $M_{j}$. Then, the model can be selected with a higher posterior probability. This method is employed to choose GARCH models by Vrontos, Dellaportas and Politis (2000), GARCH and threshold GARCH models by So et al. (2005), DTGARCH models by Chen et al. (2005) and various nonnested asymmetric heteroscedastic models by Chen et al. (2006). Using the method of RJMCMC, So, Chen, Lee, and Chang (2008) compare the two most commonly used statistical distributions in empirical analysis to capture the features of the student-t distribution and the generalized error distribution (GED).

Direct Estimation Method: An approximate estimation of model posterior probability proposed by Congdon $(2006,2007)$ is a direct estimation method that uses separate MCMC samples of $m$ competing models. An approximate Monte Carlo estimate of model posterior probability, $p\left(M_{i} \mid \boldsymbol{y}^{1, n}\right)$, is obtained as

$$
\operatorname{Pr}\left(M_{i} \mid \boldsymbol{y}^{1, n}\right) \approx \frac{1}{N-M} \sum_{j=M+1}^{N} \operatorname{Pr}\left(M_{i} \mid \boldsymbol{y}^{1, n}, \boldsymbol{\theta}_{i}^{(j)}\right),
$$

where

$$
\begin{aligned}
\operatorname{Pr} & \left(M_{i} \mid \boldsymbol{y}^{1, n}, \boldsymbol{\theta}_{i}^{(j)}\right) \\
\quad & \propto p\left(\boldsymbol{y}^{1, n} \mid \boldsymbol{\theta}_{i}^{(j)}, M_{i}\right) p\left(\boldsymbol{\theta}_{i}^{(j)} \mid M_{i}\right) \operatorname{Pr}\left(M_{i}\right)=G_{i}^{(j)},
\end{aligned}
$$

where $\boldsymbol{\theta}_{i}^{(j)}$ is the $j$ th MCMC iterate from the posterior distribution of model $M_{i}$. The normalizing factor in (8) is the sum of the terms $G_{k}^{(j)}, k=1, \ldots, m$. Thus, $\operatorname{Pr}\left(M_{i} \mid \boldsymbol{y}^{1, n}, \boldsymbol{\theta}_{i}^{(j)}\right)$ is estimated by

$$
\hat{\operatorname{Pr}}\left(M_{i} \mid \boldsymbol{y}^{1, n}, \boldsymbol{\theta}^{(j)}\right)=G_{i}^{(j)} / \sum_{k=1}^{m} G_{k}^{(j)}
$$

and

$$
\hat{\operatorname{Pr}}\left(M_{i} \mid \boldsymbol{y}^{1, n}\right) \approx \frac{1}{N-M} \sum_{j=M+1}^{N} \hat{\operatorname{Pr}}\left(M_{i} \mid \boldsymbol{y}^{1, n}, \boldsymbol{\theta}_{i}^{(j)}\right)
$$

According to this approach, the best model, that with the highest posterior model probability, is selected.

There are further assumptions required for using this direct estimation method. For discussions on these assumptions, see Congdon (2006) and Chen et al. (2008). Some results support that the approximation seems quite precise and powerful for model selection. For example, Gerlach and Chen (2008) consider the comparison of smooth transition GARCH, DTGARCH and linear GARCH models; Chen et al. (2008) select the model from various heteroscedastic models; Chen, Gerlach, and Lin (2010) compare multi-regime threshold heteroscedastic models; and Chen, Gerlach, and Lin (2011) evaluate the performance of correct selection of multi-regime capital asset pricing models.

Stochastic Search Variable Selection (SSVS): Proposed by George and McCulloch (1993), the idea of the stochastic search variable selection (SSVS) procedure is to develop a procedure that uses probabilistic considerations for selecting promising subsets of the model. An introduction of a mixture of two normals prior for the coefficients of predictors is a key point of the SSVS method. For the coefficient $\phi_{i}, i=1, \ldots, p$, where $p$ is the number of predictors, the prior is set as

$$
\phi_{i} \mid s_{i} \sim\left(1-s_{i}\right) \mathrm{N}\left(0, \tau_{i}^{2}\right)+s_{i} \mathrm{~N}\left(0, c_{i}^{2} \tau_{i}^{2}\right),
$$

where $s_{i}$ is a latent indicator variable with $s_{i}=0$ or 1 and $\operatorname{Pr}\left(s_{i}=1\right)=\pi_{i} . s_{i}$ has the dual role of indicating which normal prior applies to $\phi_{i}$ and indicating whether $\left(s_{i}=1\right)$ or not $\left(s_{i}=0\right) \phi_{i}$ is in the current model subset. The series $s_{i}$ is thus an unknown latent indicator series to be estimated or inferred, and each MCMC sample from the vector of $s_{i}$ 's, $i=1, \ldots, p$, uniquely identifies a specific model. 
In the SSVS method, the hyper-parameter settings of $\tau_{i}$ and $c_{i}$ are important since model selection results can be sensitive to change in these settings. The general criteria for setting $c_{i}$ and $\tau_{i}$ are $c_{i}>1$ and sufficiently large, and $\tau_{i}^{2}$ small and close to 0 to make $c_{i}^{2} \tau_{i}^{2}$ substantially greater than $\tau_{i}^{2}$. Chen (1999) and So and Chen (2003) adopt this idea for selecting AR and TAR models, respectively. So, Chen and Liu (2006) propose optimal subset selection among autoregressive models with exogenous variables and heteroscedastic errors, extending the idea of the SSVS procedure. In the subset selection of threshold models, Chen, Liu and Gerlach (2011) propose a sampling mechanism for subset selection among a general family of TARMA models. Incorporating the idea of Bayesian model averaging, they modify the SSVS method to consider the subset selection among more than 16 million possible subsets.

\subsection{Diagnostic checking}

Tests of Serial Independence: For checking the model adequacy, the frequentist approach is to test the serial independence of residuals. However, testing serial independence is challenging because it requires evaluation of all possible relations between the variable of interest and its lagged variables. In practice, diagnostic tests of serial independence typically focus on certain aspects of the data, such as serial correlations or ARCH-type dependence (i.e., squared correlations). McLeod and $\mathrm{Li}$ (1983) suggest testing whether the first $m$ autocorrelations of $y_{t}^{2}$ are zero using a $Q$ test. Li (2004) focuses mainly on diagnostic checks for stationary time series.

In the typical frequentist approach for parameter estimation, residual autocorrelation is a standard device for checking time series model adequacy. An important overall test of fit in ARMA models is considered by Box and Pierce (1970) with modification by Ljung and Box (1978). Li (1992) obtains the asymptotic distribution of residual autocorrelations for a general nonlinear time series. This includes the threshold model as a special case. Li and Mak (1994) obtain the asymptotic distribution of squared residual autocorrelations for a general conditional heteroscedastic nonlinear time series model. In threshold ARCH modelling, $\mathrm{Li}$ and $\mathrm{Li}$ (1996) follow the idea of $\mathrm{Li}$ and Mak (1994) to derive the asymptotic standard errors of residual autocorrelations and squared residual autocorrelations of the DTARCH models for model diagnostic checks.

Based on the MCMC methods for statistical inference, it is natural to consider a Bayesian approach for performing diagnostic checking. Chen and So (2006) and Chen, Gerlach, and Lin (2010) perform diagnostic checking by using the method in Gerlach et al. (1999) that is demonstrated to be very effective in financial time series. Based on the following time series

$$
u_{t}=F\left(y_{t} \mid \boldsymbol{y}^{1, t-1}\right), \quad t=1, \ldots, n,
$$

where $F($.$) is the cumulative distribution function. Gerlach$ et al. (1999) show that for $k \geq t$, the estimator,

$$
\hat{u}_{t}=\frac{\sum_{i=1}^{N} F\left(y_{t} \mid \boldsymbol{y}^{1, t-1}, \boldsymbol{\theta}_{k}^{(i)}\right) / p\left(\boldsymbol{y}^{t, k} \mid \boldsymbol{y}^{1, t-1}, \boldsymbol{\theta}_{k}^{(i)}\right)}{\sum_{i=1}^{N} 1 / p\left(\boldsymbol{y}^{t, k} \mid \boldsymbol{y}^{1, t-1}, \boldsymbol{\theta}_{k}^{(i)}\right)}
$$

converges to $u_{t}$ as $N \rightarrow \infty$. Here $\boldsymbol{\theta}_{k}^{(i)}$ is the $i$ th MCMC iterate sampled from the posterior given the data $\boldsymbol{y}^{1, k}$. The parameters are numerically integrated out by MCMC sampling. Based on the convergence properties of $\hat{u}_{t}$, the estimates $\hat{v}_{t}=\Phi^{-1}\left(\hat{u}_{t}\right)$ are approximately i.i.d. $N(0,1)$ under the correct model. Standard diagnostic tests can be applied to this generalized residual series, such as the Ljung-Box test for autocorrelation or studentized range test for distributional fit.

\section{FINANCIAL APPLICATIONS}

Several threshold models that lead to more flexibility in financial applications have been proposed (see Chen et al. 2005, Chen, Yang, Gerlach, and Lo 2006 and Chen and Yu 2005). Chan, Wong and Tong (2004) introduce nonlinear time series modelling techniques through the examples of actuarial science. They discuss the uses of the basic SETAR models, SETAR models with heteroscedasticity, and multivariate SETAR models in pricing insurance products. Chen et al. (2005) present clear evidence that the double threshold GARCH model, with local news threshold, outperforms the symmetric GARCH model for the G7 stock markets. The interactive effects between US and local news are observed, and an asymmetric pattern in the relationship between US and local markets is also found. Chiang, Chen and So (2007) employ a DT-GARCH model with a compositenews variable to investigate four international return series. Their model shows the evidence of asymmetric effects in both the mean and conditional variance and indicates that the volatility and persistence of bad news are greater than those of good news. So et al. (2007) propose a DT-GARCH model and incorporate the changes in log volumes in regime switching and asymmetric effects on the volatility equations. Using the posterior odds ratios for model selection, So et al. (2007) select a model that involves volumes as the best model.

Chen, Gerlach and Lin (2008) build a threshold heteroscedastic model that allows the intra-day high and low price range to depend nonlinearly on either past information or on an exogenous variable such as US market information. This model brings greater integrity to volatility estimation. Chen, Liu and So (2008) adopt the Bayesian approach to estimate model parameters and forecast valueat-risk (VaR) by the generalized THSV models. In recent years, VaR has become a standard tool for market risk measurement. For better VaR estimation, Engle and Manganelli (2004) introduce the conditional autoregressive value-at-risk (CAViaR) model to estimate the VaR directly by quantile 
regression. Some well-known dynamic conditional autoregressive quantile models are generalized in Gerlach, Chen and Chan (2011) to fully consider the nonlinear threshold family of dynamic models. This paper investigates a Bayesian solution to the general quantile regression problem via the Skewed-Laplace distribution, which is adapted and designed for parameter estimation in this model family through an adaptive MCMC sampling scheme. The threshold CAViaR (TCAV) model is as follows.

$$
f_{t}(\boldsymbol{\beta})= \begin{cases}\beta_{1}+\beta_{2} f_{t-1}(\boldsymbol{\beta})+\beta_{3}\left|y_{t-1}\right|, & z_{t-1} \leq \gamma \\ \beta_{4}+\beta_{5} f_{t-1}(\boldsymbol{\beta})+\beta_{6}\left|y_{t-1}\right|, & z_{t-1}>\gamma\end{cases}
$$

where $z_{t}$ is an observed threshold variable, which can be exogenous or self-exciting, that is, $z_{t}=y_{t}$, and $\gamma$ is the threshold value. Gerlach et al. (2010) fix $\gamma=0$, which makes TCAV a direct extension of the asymmetric slope (AS) model in Engle and Manganelli (2004). Yu, Li and Jin (2010) extend the CAViaR model by using two approaches, namely the threshold and mixture type of indirect-VaR models. The threshold type of indirect-VaR models is given by

$$
f_{t}(\boldsymbol{\beta})= \begin{cases}\left(\beta_{1}+\beta_{2} f_{t-1}^{2}(\boldsymbol{\beta})+\beta_{3} y_{t-1}^{2}\right)^{1 / 2}, & \text { if } z_{t-1}<\gamma \\ \left(\beta_{4}+\beta_{5} f_{t-1}^{2}(\boldsymbol{\beta})+\beta_{6} y_{t-1}^{2}\right)^{1 / 2}, & \text { if } z_{t-1} \geq \gamma\end{cases}
$$

Chen, Gerlach, Lin and Lee (2011) assess the possibility of general Bayesian forecasting for carrying out one- to tenday ahead VaR across a range of competing parametric heteroscedastic models.

While threshold modelling is widely applied in financial time series as mentioned above, several relevant issues are considered under the threshold models, such as threshold unit root tests and threshold co-integration. The threshold unit root problem is first considered by Pham, Chan and Tong (1991) as a nonlinear unit root problem. They show that the least squares estimators (LSE) of the parameters are strongly consistent under a simplified TAR(1) model. Using the SETAR models of Tong (1990), Kräger and Kugler (1993) propose the modelling of nonlinear dependencies in the conditional mean of exchange rate changes. Their results indicate that moderate or large, negative or positive exchange rate changes follow different AR behaviors. Bec, Salem and Carrasco (2004) consider modelling the real exchange rate by a stationary three-regime SETAR model with the possibility of a unit root in the middle regime. This representation is consistent with purchasing power parity in the presence of trading costs. Their main contribution is to provide statistical tools for testing the unit root versus a SETAR. In some cases, results reject the null hypothesis of a linear unit root in favour of a stationary three-regime SETAR model, e.g., Taylor (2001).

In 1982, Granger introduces the concept of cointegration, which means integrated series for which a linear combination exists that is stationary.

$$
\beta_{1} Y_{t 1}+\beta_{2} Y_{t 2}+\cdots+\beta_{K} Y_{t K}=Z_{t}
$$

where $Z_{t}$ is a stationary series. This can be interpreted economically as the presence of long-run equilibrium, the relationship between the variables being stable. That is, cointegration is useful since it identifies a long-run relationship between I(1) variables. Granger's representation theorem states that if a set of variables is cointegrated then there always exists an error-correcting formulation of the dynamic model and vice versa. Motivated by the TAR model of Tong (1978, 1983), Balke and Fomby (1997) introduce the concept of threshold cointegration, which allows us to take into account the main criticisms raised against linear cointegration. In their setup, the adjustment does not need to occur instantaneously but only once the deviations exceed some critical threshold, allowing the presence of an inaction or no-arbitrage band. Hence, the linear adjustment process is extended to

$$
z_{t}= \begin{cases}\rho_{L} z_{t-1}+u_{t}, & \text { if } z_{t-1} \leq \theta_{L} \\ \rho_{M} z_{t-1}+u_{t}, & \text { if } \theta_{L} \leq z_{t-1} \leq \theta_{H} \\ \rho_{H} z_{t-1}+u_{t}, & \text { if } \theta_{H} \leq z_{t-1},\end{cases}
$$

where autoregressive parameters are denoted by subscripts $L, M$, and $H$, standing for low, middle, and high regimes, respectively. While the work of Balke and Fomby (1997) focuses on the long-run relationship representation, extension to a threshold vector error correction model has been made by several authors, e.g., Granger and Lee (1989), Hansen and Seo (2002), Lo and Zivot (2001), etc. Kapetanios and Shin (2006) propose a testing procedure to distinguish a unit root process from a globally stationary three-regime self-exciting threshold autoregressive process.

\section{CONCLUSIONS}

Nowadays, threshold time series models and threshold philosophy are successfully applied to many practical problems in finance. The Bayesian paradigm provides a rich framework for inference and decision making with modern heteroscedastic models. Looking ahead, it seems that the threshold principle will continue to make worthwhile contributions to financial time series analysis. In particular, it will lead to advances in multivariate time series modelling, panel time series modelling, and others.

\section{APPENDIX}

\section{Double smooth-transition GARCH Model.}

A time series $\left\{y_{t}\right\}$ is a Double Smooth-Transition GARCH (DST) process, if it satisfies,

$$
\begin{aligned}
& y_{t}=\mu_{t}^{(1)}+G\left(z_{t-d} ; \gamma, c\right) \mu_{t}^{(2)}+a_{t}, \\
& a_{t}=\sqrt{h_{t}} \varepsilon_{t}, \quad \varepsilon_{t} \sim D(0,1), \\
& h_{t}=h_{t}^{(1)}+G\left(z_{t-d} ; \gamma, c\right) h_{t}^{(2)},
\end{aligned}
$$




$$
\begin{aligned}
& \mu_{t}^{(l)}=\phi_{0}^{(l)}+\sum_{i=1}^{p} \phi_{i}^{(l)} y_{t-i}+\sum_{j=1}^{q} \psi_{j}^{(l)} x_{t-j}, \\
& h_{t}^{(l)}=\alpha_{0}^{(l)}+\sum_{i=1}^{g} \alpha_{i}^{(l)} a_{t-i}^{2}+\sum_{j=1}^{k} \beta_{j}^{(l)} h_{t-j}, \quad l=1,2,
\end{aligned}
$$

$$
s_{t}= \begin{cases}0 & \text { if } z_{t-d}<r \\ 1 & \text { if } z_{t-d} \geq r\end{cases}
$$

with the threshold value $r$ and the delay $d$ of threshold variable $z_{t}$ both considered unknown parameters.

\section{ACKNOWLEDGEMENTS}

where $D(0,1)$ is a distribution with mean 0 and variance 1 and the $G($.$) is a smooth-transition function. In practice,$ $G($.$) often assumes one of three forms - namely, a logis-$ tic, exponential, or cumulative distribution function. Two commonly used transition functions are the logistic and exponential, which are given below.

$$
\begin{aligned}
G\left(z_{t-d} ; \gamma, c\right) & =\frac{1}{1+\exp \left\{-\gamma\left(\frac{z_{t-d}-c}{s_{z}}\right)\right\}}, \\
G^{*}\left(z_{t-d} ; \gamma, c\right) & =1-\exp \left\{-\gamma\left|\frac{z_{t-d}-c}{s_{z}}\right|\right\} \\
& \text { or } 1-\exp \left\{-\gamma\left(\frac{z_{t-d}-c}{s_{z}}\right)^{2}\right\},
\end{aligned}
$$

where $\gamma$ is the smoothness of change in the value of the function, usually assumed positive; $c$ is the threshold value and $s_{z}$ is the sample standard deviation of the observed threshold variable $z_{t}$; this allows $\gamma$ to be scale-free and therefore comparable across different return series. A full Bayesian inference procedure is developed by Gerlach and Chen (2008) to examine whether asymmetry in time series is better modelled by a logistic transition function in both mean and volatility equations. Chen, Gerlach, Choy and Lin (2010) apply DST-GARCH with an exponential smooth transition to financial data and highlight the presence of both mean and volatility (size) asymmetry.

\section{Threshold stochastic volatility (THSV) Model.}

The generalized THSV model in Chen, Liu and So (2008) is defined as

$$
\begin{aligned}
& R_{t}=\left(\psi_{0}+\delta_{0} s_{t}\right)+\sum_{i=1}^{p}\left(\psi_{i}+\delta_{i} s_{t}\right) R_{t-i} \\
&+\sum_{k=1}^{l} \sum_{j=1}^{q_{k}}\left(\phi_{k, j}+\gamma_{k, j} s_{t}\right) x_{k, t-j}+a_{t}, \\
& a_{t}= e^{h_{t} / 2} u_{t}, \quad u_{t} \sim \mathrm{t}_{\nu}(0,1) \\
& h_{t+1}=\left(\alpha_{0}+\beta_{0} s_{t+1}\right)+\sum_{k=1}^{g}\left(\alpha_{k}+\beta_{k} s_{t+1}\right) h_{t+1-k}+\eta_{t}, \\
& \eta_{t} \sim \mathrm{N}\left(0, \sigma^{2}\right)
\end{aligned}
$$

where $p$ and $g$ are the orders of the autoregressive processes of mean and variance equations, $l$ is the number of exogenous variables, and $q_{k}$ is the lag of the $k$ th exogenous variable. The state variable is defined as
We thank the editor and an anonymous referee for their insightful and helpful comments and suggestions, which improved this paper. Cathy Chen is supported by the grant: 99-2118-M-035-001-MY2 from the National Science Council (NSC) of Taiwan.

Received 10 September 2010

\section{REFERENCES}

Akaike, H. (1974) A new look at the statistical model identification. IEEE Transactions on Automatic Control, AC-19, 716-723. MR0423716

Amendola, A., Niglio, M. and Vitale, C. (2006) The moments of SETARMA models. Statistics \& Probability Letters, 76, 625-633. MR2255792

Anderson, H. M., NAm, K. and Vahid, F. (1999) Asymmetric nonlinear smooth transition GARCH models. In P. Rothman (ed.), Nonlinear Time Series Analysis of Economic and Financial Data, Kluwer, Boston, 191-207. MR2478830

Asai, M. and McAleer, M. (2006) Asymmetric multivariate stochastic volatility. Econometric Reviews, 25, 453-473. MR2256294

Audrino, F. and Bühlmann, P. (2001) Tree-structured GARCH models. Journal of the Royal Statistical Society, Series B, 63, 727-744. MR1872063

Audrino, F. and Trojani, F. (2006) Estimating and predicting multivariate volatility thresholds in global stock markets. Journal of Applied Econometrics, 21, 345-369. MR2224238

BACON, D. W. and WATTS, D. G. (1971) Estimating the transition between two interesting straight lines. Biometrika, 58, 525-534.

Balke, N. S. and Fomby, T. B. (1997) Threshold cointegration. International Economic Review, 38, 627-645. MR1467826

BAnZ, R. W. (1981) The relationship between return and market value of common stocks. Journal of Financial Economics, 9, 3-18.

Bauwens, L., Preminger, A. and Rombouts, J. V. K. (2010) Theory and inference for a Markov switching GARCH model. Econometrics Journal, 13, 218-244. MR2722883

Bec, F., Salem, M. B. and Carrasco, M. (2004) Tests for unit-root versus threshold specification with an application to the purchasing power parity relationship. Journal of Business and Economic Statistics, 22, 382-395. MR2091567

BLACK, F. (1976) The pricing of commodity contracts. Journal of Financial Economics, 3, 167-179.

Boeroa, G. and Marrocu, E. (2004) The performance of SETAR models: A regime conditional evaluation of point, interval and density forecasts. International Journal of Forecasting, 20, 305-320.

Bollerslev, T. (1986) Generalized autoregressive conditional heteroskedasticity. Journal of Econometrics, 31, 307-327. MR0853051

Bollerslev, T., Engle, R. F. and Wooldridge, J. M. (1988) A capital asset pricing model with time-varying covariances. Journal of Political Economy, 96, 116-131.

Box, G. E. P. and Pierce, D. A. (1970) Distribution of the autocorrelations in autoregressive moving average time series models. Journal of the American Statistical Association, 65, 1509-1526. MR0273762

Brockwell, P., Liu, J. and Tweedie, R. L. (1992) On the existence of stationary threshold autoregressive moving-average processes. Journal of Time Series Analysis, 13, 95-107. MR1165659 
Brooks, C. (2001) A double-threshold GARCH model for the French Franc/Deutschmark exchange rate, Journal of Forecasting, 20, 135143.

Brooks, C. and Garrett, I. (2002) Can we explain the dynamics of the UK FTSE 100 stock and stock index futures markets. Applied Financial Economics, 12, 25-31.

CAporin, C. and McAleer, M. (2006) Thresholds, news impact surfaces and dynamic asymmetric multivariate GARCH. Working paper.

Chan, K. S. (1990) Testing for threshold autoregression. Annals of Statistics, 18, 1886-1894. MR1074443

Chan, K. S. (1993) Consistency and limiting distribution of the least squares estimator of a threshold autoregressive model. The Annals of Statistics, 21, 520-533. MR1212191

Chan, K. S. (2009) Exploration of a Nonlinear World, 29, World Scientific, Singapore.

Chan, K. S., Petruccelli, J. D., Tong, H. and Woolford, S. W (1985) A multiple-threshold AR(1) model. Journal of Applied Probability, 22, 267-279. MR0789351

Chan, K. S. and Tong, H. (1985) On the use of deterministic Lyapunov function for the ergodicity of stochastic difference equations. Advances in Applied Probability, 17, 667-678. MR0798881

Chan, K. S. and Tong, H. (1986) On estimating thresholds in autoregressive models. Journal of Time Series Analysis, 7, 179-190. MR0857248

Chan, W. S., Wong, A. C. S. and Tong, H. (2004) Some nonlinear threshold autoregressive time series models for actuarial use. North American Actuarial Journal, 8, 37-61. MR2100929

Chen, C. W. S. (1998) A Bayesian analysis of generalized threshold autoregressive models. Statistics \& Probability Letters, 40, 15-22. MR1650569

Chen, C. W. S. (1999) Subset selection of autoregressive time series models. Journal of Forecasting, 18, 505-516.

Chen, C. W. S., Chiang, T. C. and So, M. K. P. (2003) Asymmetrical reaction to US stock-return news: Evidence from major stock markets based on a double-threshold model, The Journal of Economics and Business, 55, 487-502.

Chen, C. W. S., Gerlach, R., Choy, B. and Lin, C. (2010) Estimation and inference for exponential smooth transition nonlinear volatility models. Journal of Statistical Planning and Inference, 140, 719-733. MR2558400

Chen, C. W. S., Gerlach, R. and Lin, A. M. H. (2010) Falling and explosive, dormant and rising markets via multiple-regime financial time series models. Applied Stochastic Models in Business and Industry, 26, 28-49. MR2663061

Chen, C. W. S., Gerlach, R. and Lin, A. M. H. (2011) Multi-regime nonlinear capital asset pricing models. Quantitative Finance, DOI: 10.1080/14697680902968013.

Chen, C. W. S., Gerlach, R. and Lin, E. M. H. (2008) Volatility forecast using threshold heteroskedastic models of the intra-day range. Computational Statistics and Data Analysis, on Statistical \& Computational Methods in Finance, 52, 2990-3010. MR2424772

Chen, C. W. S., Gerlach, R., Lin, E. M. H. and Lee, W. (2011) Bayesian forecasting for financial risk management, pre and post the global financial crisis, Journal of Forecasting, DOI: 10.1002/for.1237.

Chen, C. W. S., Gerlach, R. H. and So, M. K. P. (2006) Comparison of nonnested asymmetric heteroscedastic models. Computational Statistics 85 Data Analysis, a special issue on Nonlinear Modelling and Financial Econometrics, 51, 2164-2178. MR2307493

Chen, C. W. S., Gerlach, R. and So, M. K. P. (2008) Bayesian model selection for heteroskedastic models, Advances in Econometrics, 23 Special Issue on Bayesian Econometrics, 567-594.

Chen C. W. S., Gerlach, R. and Tai, A. P. J. (2008) Testing for nonlinearity in mean and volatility for heteroskedastic models, Mathematics and Computers in Simulation, 79, 489-499. MR2477542

Chen, C. W. S. and Lee, J. C. (1995) Bayesian inference of threshold autoregressive models. Journal of Time Series Analysis, 16, 483492. MR1365643
Chen, C. W. S., Lin, E. M. H., Liu, F. C. and Gerlach, R. (2008) Bayesian estimation for parsimonious threshold autoregressive models in R. The R Journal, 8, 26-33.

Chen, C. W. S., Lin, S. and Yu, P. L. H. (2011) Smooth transition quantile capital asset pricing models with heteroscedasticity. Computational Economics, DOI 10.1007/s10614-011-9266-y.

Chen, C. W. S., Liu, F. C. and Gerlach, R. (2011) Bayesian subset selection for threshold autoregressive moving-average models. Computational Statistics, 26, 1-30.

Chen, C. W. S., Liu, F. C. and So, M. K. P. (2008) Heavy-tailed distributed threshold stochastic volatility models in financial time series. Australian \& New Zealand Journal of Statistics, 50, 29-51. MR2414654

Chen, C. W. S. and So, M. K. P. (2006) On a threshold heteroscedastic model, International Journal of Forecasting, 22, 73-89.

Chen, C. W. S., So, M. K. P. and Gerlach, R. H. (2005) Assessing and testing for threshold nonlinearity in stock returns, Australian \&S New Zealand Journal of Statistics, 47, 473-488. MR2235417

Chen, C. W. S., So, M. K. P. and Lin, E. M. H. (2009) Volatility forecasting with double Markov switching GARCH models. Journal of Forecasting, 28, 681-697. MR2756466

Chen, C. W. S., Yang, M. J., Gerlach, R. H. and Lo, H. J. (2006) The asymmetric reactions of mean and volatility of stock returns to domestic and international information based on a four-regime double-threshold GARCH model. Physica A - Statistical Mechanics and Its Applications, 366, 401-418.

Chen, C. W. S. and YU, T. H. K. (2005) Long-term dependence with asymmetric conditional heteroscedasticity in stock returns. Physica A - Statistical Mechanics and Its Applications, 353, 413-424.

Chen, R. (1995) Threshold variable selection in open-loop threshold autoregressive models. Journal of Time Series Analysis, 16, 461481. MR1365642

Chen, R. and Tsay, R. S. (1991) On the ergodicity of TAR(1) processes. Annals of Applied Probability, 1, 613-634. MR1129777

Chinang, T. C., Chen, C. W. S. and So, M. K. P. (2007) Asymmetric return and volatility responses to composite news from stock markets. Multinational Finance Journal, 11, 179-210.

Chib, S., Nardari, F. and Shephard N. (2006) Analysis of high dimensional multivariate stochastic volatility models. Journal of Econometrics, 134, 341-371. MR2328413

Christie, A. A. (1982) The stochastic behavior of common stock variances: Value, leverage and interest rate effects. Journal of Financial Economics, 10, 407-432.

Congdon, P. (2006) Bayesian model choice based on Monte Carlo estimates of posterior model probabilities. Computational Statistics E Data Analysis, 50, 346-357. MR2201867

Congdon, P. (2007) Model weights for model choice and averaging. Statistical Methodology, 4, 143-157. MR2368141

De Goeis, P. and Marquering, W. (2004) Modeling the conditional covariance between stock and bond returns: A multivariate garch approach. Journal of Financial Econometrics, 2, 531-564.

De GooiJer, J. G. (1998) On threshold moving-average models. Journal of Time Series Analysis, 19, 1-18. MR1624163

ENGLE, R. F. (1982) Autoregressive conditional heteroscedasticity with estimates of the variance of United Kingdom inflation. Econometrica, 50, 987-1008. MR0666121

Engle, R. F. (2002) Dynamic conditional correlation: A simple class of multivariate generalized autoregressive conditional heteroskedasticity models. Journal of Business and Economic Statistics, 20, 339-350. MR1939905

Engle, R. F. and Manganelli, S. (2004) CAViaR: Conditional autoregressive value at risk by regression quantiles. Journal of Business and Economic Statistics, 22, 367-381. MR2091566

Engle, R. F. and NG, V. K. (1993) Measuring and testing the impact of news on volatility. Journal of Finance, 48, 1749-1778.

FAMA, E. F., FRENCH, K. R. (1992) The cross-section of expected stock returns. Journal of Finance, 47, 427-465.

Franses, P. H. and van DiJk, D. (2000) Nonlinear Time Series Models in Empirical Finance, Cambridge University Press, Cambridge. 
George, E. I. and McCulloch, R. E. (1993) Variable selection via Gibbs sampling. Journal of the American Statistical Association, 88, 881-889.

Gerlach, R., Carter, C. K. and Kohn, R. (1999) Diagnostics for time series analysis. Journal of Time Series Analysis, 20, 309-330. MR1693161

Gerlach, R. and Chen, C. W. S. (2008) Bayesian inference and model comparison for asymmetric smooth transition heteroskedastic models, Statistics and Computing, 18, 391-408. MR2461884

Gerlach, R., Chen, C. W. S. and Chan, N. C. Y. (2011) Bayesian time-varying quantile forecasting for value-at-risk in financial markets, Journal of Business and Economic Statistics, DOI: 10.1198/jbes.2010.08203.

Gerlach, R., Chen, C. W. S., Lin, D. S. Y. and Huang, M. H. (2006) Asymmetric responses of international stock markets to trading volume. Physica A - Statistical Mechanics and Its Applications, 360, 422-444.

Geweke, J. (1995) Bayesian comparison of econometric models. Working Paper 532. Research Department, Federal Reserve Bank of Minneapolis.

Ghaddar, D. K. and Tong, H. (1981) Data transformation and selfexciting threshold autoregression. Applied Statistics, 30, 238-248.

Ghysels, E. (1998) On stable factor structures in the pricing of risk: Do time-varying betas help or hurt? Journal of Finance, 53, 549573.

Glosten, L. R., Jagannathan, R. and Runkle, D. E. (1993) On the relation between the expected value and the volatility of the nominal excess return on stocks. Journal of Finance, 48, 1779-1801.

GonzÁlez-RiverA, G. (1998) Smooth-transition GARCH models. Studies in Nonlinear Dynamics and Econometrics, 3, 61-78.

Granger, C. W. J. and Lee, T. H. (1989) Investigation of production, sales and inventory relationships using multicointegration and non-symmetric error correction models. Journal of Applied Econometrics, 4, S145-S159.

Granger, C. W. J. and Teräsvirta, T. (1993) Modelling Nonlinear Economic Relationships, Oxford University Press, Oxford.

Green, P. J. (1995) Reversible jump MCMC computation and Bayesian model determination. Biometrika, 82, 711-732. MR1380810

HaGerud, G. E. (1996) A smooth transition ARCH model for asset returns. Working Paper Series in Economics and Finance No. 162, Stockholm School of Economics.

Hamilton, J. D. (1989) A new approach to the economic analysis of nonstationary time series subject to changes in regime, Econometrica, 57, 357-384. MR0996941

HANsen, B. (1997) Inference in TAR Models. Studies in Nonlinear Dynamics \& Econometrics, 2, Article 1. MR1467458

Hansen, B. and Seo, B. (2002) Testing for two-regime threshold cointegration in vector error-correction models. Journal of Econometrics, 110, 293-318. MR1928307

Harvey, A. C., Ruiz, E. and Shephard, N. (1994) Multivariate stochastic variance models, Review of Economic Studies, 61, 247264.

Kapetanios, G. and Shin, Y. (2006) Unit root tests in three-regime SETAR models. Econometrics Journal, 9, 252-278. MR2324969

Kapetanios, G., Shin, Y. and Snell, A. (2003) Testing for a unit root against Nonlinear STAR Models. Journal of Econometrics, 112, 359-379. MR1951148

KAss, R. and RAfTery, A. (1995) Bayes factors. Journal of the American Statistical Association, 90, 773-795.

Keenan, D. M. (1985) A Tukey nonadditivity-type test for time series nonlinearity. Biometrika, 72, 39-44. MR0790199

Koutmos, G. (1999) Asymmetric price and volatility adjustments in emerging Asian stock markets. Journal of Business Finance and Accounting, 26, 83-101.

KRÄGER, H. and Kugler, P. (1993) Non-linearities in foreign exchange markets: A different perspective. Journal of International Money and Finance, 12, 195-208.
Kwan, W., LI, W. K. and NG, K. W. (2010) A multivariate threshold varying conditional correlations model. Econometric Reviews, 29, $20-38$.

LI, W. K. (1992) On the asymptotic standard errors of residual autocorrelations in nonlinear time series modelling. Biometrika, $\mathbf{7 9}$, 435-437. MR1185148

LI, W. K. (2004) Diagnostic Checks in Time Series, Chapman\& Hall/CRC, Boca Raton.

LI, W. K. (2009) The threshold approach to volatility modelling. In K. S. Chan (ed.), Exploration of a nonlinear model: An appreciation of Howell Tong's contributions to statistics, World Scientific, Singapore, 95-100.

LI, C. W. and LI, W. K. (1996) On a double-threshold autoregressive heteroscedastic time series model. Journal of Applied Econometrics, 11, 253-274.

LI, W. K. and MAK, T. K. (1994) On the squared residual autocorrelations in non-linear time series with conditional heteroskedasticity. Journal of Time Series Analysis, 15, 627-636. MR1312326

LiNG, S. (1999) On the probabilistic properties of a double threshold ARMA conditional heteroskedastic model. Journal of Applied Probability, 36, 688-705. MR1737046

Ling, S. and Tong, H. (2005) Testing for a linear MA model against threshold MA models. The Annals of Statistics, 33, 2529-2552. MR2253094

Ling, S., Tong, H. and LI, D. (2007) Ergodicity and invertibility of threshold moving-average models. Bernoulli, 13, 161-168. MR2307400

Liu, L. M. (2009) Time Series and Forecasting, 2nd ed. Scientific Computing Associates Corp.

Lo, M. C. and Zıvot, E. (2001) Threshold cointegration and nonlinear adjustment to the law of one price. Macroeconomic Dynamics, 5, 533-576.

LJung, G. M. and Box, G. E. P. (1978) On a measure of lack of fit in time series models. Biometrika, 65, 297-303.

McAleer, M., Hoti, S. and Chan, F. (2009) Structure and asymptotic theory for multivariate asymmetric conditional volatility. Econometric Reviews, 28, 422-440. MR2750985

McAleer, M. and Medeiros, M. C. (2008) A multiple regime smooth transition heterogeneous autoregressive model for long memory and asymmetries. Journal of Econometrics, 147, 104-119. MR2472985

McCulloch, R. E. and Tsay, R. S. (1994) Statistical analysis of economic time series via Markov switching models. Journal of Time Series Analysis, 15, 523-539. MR1263893

McLeOD, A. I. and LI, W. K. (1983) Diagnostic checking ARMA time series models using squared-residual autocorrelations. Journal of Time Series Analysis, 4, 269-273. MR0738587

Nam, K., Pyun, C. S. and Avard, S. L. (2001) Asymmetric reverting behavior of short-horizon stock returns: An evidence of stock market overreaction. Journal of Banking \& Finance, 25, 807-824.

Nelson, D. B. (1991) Conditional heteroskedasticity in asset returns: A new approach. Econometrica, 59, 347-370. MR1097532

Pelletier, D. (2006) Regime switching for dynamic correlations. Journal of Econometrics, 131, 445-473. MR2276007

Petruccelli, J. D. and Davies, N. (1986) A portmanteau test for selfexciting threshold autoregressive-type nonlinearity in time series. Biometrika, 73, 687-694. MR0897860

Petruccelli, J. D. and Woolford, S. W. (1984) A threshold AR(1) model. Journal of Applied Probability, 21, 270-286. MR0741130

Pham, D. T., Chan, K. S. and Tong, H. (1991) Strong consistency of least squares estimator for non-ergodic threshold autoregressive model. Statistica Sinica, 1, 361-369. MR1130124

Poon, S. H. and Granger, C. W. J. (2003) Forecasting volatility in financial markets: A review. Journal of Economic Literature, 41, $478-539$.

Rabemananjara, R. and Zakoian, J. M. (1993) Threshold ARCH models and asymmetries in volatility. Journal of Applied Econometrics, 8, 31-49.

So, M. K. P. and Chen, C. W. S. (2003) Subset threshold autoregression. Journal of Forecasting, 22, 49-66. 
So, M. K. P., Chen, C. W. S. and Chen, M. T. (2005) A Bayesian threshold nonlinearity test for financial time series. Journal of Forecasting, 24, 61-75. MR2143086

So, M. K. P., Chen, C. W. S., Chiang, T. C. and Lin, D. S. Y. (2007) Modeling financial time series with threshold nonlinearity in returns and trading volume. Applied Stochastic Models in Business and Industry, 23, 319-338. MR2408671

So, M. K. P., Chen, C. W. S., Lee, J. Y. and Chang, Y. P. (2008) An empirical evaluation of fat-tailed distributions in modeling financial time series. Mathematics and Computers in Simulation, 77, 96-108. MR2388253

So, M. K. P., Chen, C. W. S. and LiU, F. C. (2006) Best subset selection of autoregressive models with exogenous variables and generalized autoregressive conditional heteroscedasticity errors. Journal of the Royal Statistical Society Series C, 55, 201-224. MR2226545

So, M. K. P. and Choi, C. Y. (2008) A multivariate threshold stochastic volatility model. Mathematics and Computers in Simulation, $\mathbf{7 9}$ 306-317. MR2477532

So, M. K. P. and CHOI, C. Y. (2009) A multivariate factor threshold stochastic volatility model. Journal of Forecasting, 28, 712-735. MR2756468

So, M. K. P., LAm, K. and Li, W. K. (1998) A stochastic volatility model with Markov switching. Journal of Business 8 Economic Statistics, 16, 244-253. MR1615375

So, M. K. P., LI, W. K. and LAM, K. (1997) Multivariate modelling of the autoregressive random variance process. Journal of Time Series Analysis, 18, 429-446. MR1466887

So, M. K. P., LI, W. K. and LAM, K. (2002) A threshold stochastic volatility model. Journal of Forecasting, 21, 473-500.

So, M. K. P. and YIP, I. W. H. (2010) A multivariate GARCH model with aggregate threshold dynamics. http://ssrn.com/abstract $=1538690$.

So, M. K. P. and YIP, I. W. H. (2011) Multivariate GARCH models with correlation clustering. Forthcoming, Journal of Forecasting.

TAYLOR, A. M. (2001) Potential pitfalls for the PPP puzzle? Sampling and specification biases in mean reversion tests of the law of one price. Econometrica, 69, 473-98.

TAYlor, S. J. (1986) Modelling Financial Time Series, John Wiley, New York.

TERÄSvirTA, T. (1994) Specification, estimation, and evaluation of smooth transition autoregressive models. Journal of the American Statistical Association, 89, 208-218.

TERÄsvirTA, T. (1998) Modelling economic relationships with smooth transition regressions. In A. Ullah and D. E. A. Giles (eds.), Handbook of Applied Economic Statistics, Marcel Dekker, New York, 507552 .

Tong, H. (1978) On a threshold model. In C. H. Chen (ed.), Pattern Recognition and Signal Processing, Sijthoff \& Noordhoff, Amsterdam. MR0552641

Tong, H. (1983) Threshold Models in Nonlinear Time Series Analysis. Lecture Notes in Statistics, Springer-Verlag, New York. MR0717388

Tong, H. (1990) Non-linear Time Series: A Dynamical System Approach. Clarendon Press, Oxford. MR1079320

Tong, H. and LIM, K. S. (1980) Threshold autoregression, limit cycles and cyclical data (with discussion). Journal of the Royal Statistical Society: Series B, 42, 245-292.

TsAY, R. S. (1986) Nonlinearity tests for time series. Biometrika, 73, 461-466. MR0855906

TsAY, R. S. (1989) Testing and modeling threshold autoregressive processes. Journal of the American Statistical Association, 84, 231240. MR0999683

TsAY, R. S. (1998) Testing and modeling multivariate threshold models. Journal of the American Statistical Association, 93, 1188-1202. MR1649212
TsE, Y. K. and Tsui, A. K. C. (2002) A multivariate generalized autoregressive conditional heteroscedasticity model with time-varying correlations. Journal of Business and Economic Statistics, 20, 351361. MR1939906

Tukey, J. W. (1949) One degree of freedom for non-additivity. Biometrics, 5, 232-242.

Tyssedal, J. S. and TJøstheim, D. (1988) An autoregressive model with suddenly changing parameters and an application to stock market prices. Applied Statistics, 37, 353-369. MR0966805

van Dijk, D., Teräsvirta, T. and Franses, P. H. (2002) Smooth transition autoregressive models - A survey of recent developments. Econometric Reviews, 21, 1-47. MR1893981

Vrontos, I., Dellaportas, P. and Politis, D. (2000) Full Bayesian inference for GARCH and EGARCH models. Journal of Business and Economic Statistics, 18, 187-198.

Wong, C. S., Chan, W. S. and Kam, P. L. (2009) A student tmixture autoregressive model with applications to heavy-tailed financial data. Biometrika, 96, 751-760. MR2538770

WonG, C. S. and LI, W. K. (1997) Testing for threshold autoregression with conditional heteroscedasticity. Biometrika, 84, 407-418. MR1467056

Wong, C. S. and LI, W. K. (2000a) On a mixture autoregressive model. Journal of the Royal Statistical Society, Series B, 62, 95115. MR1747398

Wong, C. S. and LI, W. K. (2000b) Testing for double threshold autoregressive conditional heteroscedastic model. Statistica Sinica, 10, 173-189. MR1742107

Wong, C. S. and LI, W. K. (2001) On a mixture autoregressive conditional heteroscedastic model. Journal of the American Statistical Association, 96, 982-995. MR1946366

Wu, S. and Chen, R. (2007) Threshold variable determination and threshold variable driven switching autoregressive models. Statistica Sinica, 17, 241-264.

YU, P. L. H., LI, W. K. and JIN, S. (2010) On some models for valueat-risk. Econometric Reviews, 29, 622-641. MR2747264

ZakoiAn, J. M. (1994) Threshold heteroskedastic models. Journal of Economic Dynamics and Control, 18, 931-955.

Cathy W. S. Chen

Department of Statistics

Feng Chia University, Taichung

Taiwan

E-mail address: chenws@mail.fcu.edu.tw

Mike K. P. So

Department of Information Systems

Business Statistics and Operations Management

The Hong Kong University of Science \& Technology

Clear Water Bay

Hong Kong

E-mail address: immkpso@ust.hk

Feng-Chi Liu

Department of Statistics

Feng Chia University, Taichung

Taiwan

E-mail address: lifanliu1024@gmail.com 